\title{
ARTÍCULO ORIGINAL \\ Estrés, ansiedad y factores asociados en mujeres adolescentes embarazadas y no embarazadas en Medellín (Colombia)
}

Fecha de recibido:

18 de mayo de 2020.

Fecha de aprobación:

29 de septiembre de 2020 .
Forma de citar este artículo: Bonilla-Sepúlveda OA. Estrés, ansiedad y factores asociados en mujeres adolescentes embarazadas y no embarazadas en Medellín (Colombia). Med UPB. 2021;40(1):2-9. DOI:10.18566/medupb.v40n1.a02

1 Grupo de investigación en cáncer IDC. Medellín, Colombia.

2 UniRemington. Medellín, Colombia.

Dirección de correspondencia:

Óscar Alejandro Bonilla Sepúlveda. Correo electrónico: mastologia. bonilla@gmail.com
Stress, anxiety, and associated factors in pregnant and nonpregnant adolescent women in Medellin (Colombia) / Estresse, ansiedade e fatores associados em adolescentes grávidas e não grávidas em Medellín (Colômbia)

Óscar Alejandro Bonilla-Sepúlveda ${ }^{1,2}$

\section{RESUMEN}

Objetivo: Comparar el grado de ansiedad y estrés en dos grupos poblacionales de mujeres adolescentes y su asociación con el embarazo.

Metodología: Estudio transversal analítico en 125 adolescentes embarazadas atendidas en el Hospital General de Medellín (Colombia) y 124 adolescentes no embarazadas estudiantes del Colegio Jorge Eliécer Gaitán de Medellín (Colombia). Las participantes respondieron la encuesta de Hamilton para ansiedad y de Holmes y Rahe para estrés psicosocial.

Resultados: La mediana de edad fue 17 años (rango intercuartil o RIC=2) en las embarazadas y $13.5(\mathrm{RIC}=2)$ en las estudiantes. En las embarazadas, la prevalencia de ansiedad fue del $68 \%$ y de estrés del $73.6 \%$, mientras que en el grupo de no embarazadas la prevalencia de ansiedad fue del $64 \%(O R=1.2$. IC95\% 0.20-2.09. $p=0.5)$ y la de estrés fue del 40\% (OR=4.18; IC95\% 2.37-7.41; $\mathrm{p}=<0.01)$. Hubo asociación entre tener una prueba positiva para ansiedad y malas relaciones familiares, consumo de alcohol e inicio de vida sexual, mientras que hubo asociación entre test positivo para estrés y ser menor de 15 años, tener malas relaciones familiares, malas relaciones de pareja, consumo de alcohol, inicio de vida sexual y familiares con enfermedad mental.

Conclusiones: No hay mayor prevalencia de ansiedad en las madres adolescentes atendidas en el Hospital General de Medellín en comparación con las adolescentes no embarazadas del Colegio Jorge Eliécer Gaitán de Medellín (Colombia). Se encontró más estrés entre las adolescentes embarazadas. Se evidenciaron como factores asociados las malas relaciones familiares, el consumo de alcohol y el inicio de vida sexual, tanto para la ansiedad como para el estrés.

Palabras clave: adolescente; ansiedad; estrés psicológico; embarazo en adolescencia

\section{ABSTRACT}

Objective: To compare the levels of anxiety and stress in two population groups of adolescent women and their relation to pregnancy.

Methodology: Descriptive cross-sectional study, which included 125 pregnant adolescents treated at the Luz Castro de Gutiérrez Hospital, and 124 non-pregnant teenage students belonging to the Jorge Eliécer Gaitán school, both located in the city of Medellín (Colombia). The participants answered the Hamilton Anxiety Rating Scale (HAM-A) and the Holmes and Rahe Stress Scale to assess psychosocial stress.

Results: The median age was 17 years (interquartile range or IQR = 2) in pregnant women and 13.5 (IQR = 2) in students. In pregnant women, the prevalence of anxiety was $68 \%$ and stress was $73.6 \%$, while in the non-pregnant group the prevalence of anxiety was 
$64 \%(\mathrm{OR}=1.2 .95 \% \mathrm{CI} 0.20-2.09 . \mathrm{P}=0.5)$ and the stress rate was $40 \%(\mathrm{OR}=4.18 ; 95 \%$ CI $2.37-7.41 ; \mathrm{p}=\langle 0.01$ ). An association was found between having a positive test for anxiety and bad family relationships, alcohol consumption and the beginning of sexual life, while there was an association between having a positive test for stress and being under 15 years old, having bad family relationships, bad relationships with a partner, consumption of alcohol, beginning of sexual life and relatives with mental illnesses.

Conclusions: No higher prevalence of anxiety is found in adolescent mothers treated at the Hospital General de Medellín compared to non-pregnant girls from Colegio Jorge Eliécer Gaitán in Medellín (Colombia). A higher stress disorder was found among pregnant teens. Poor family relationships, alcohol consumption and the onset of sexual life were evidenced as associated factors, both for anxiety and for stress.

Keywords: adolescent; anxiety; psychological stress; pregnancy in adolescence

\section{RESUMO}

Objetivo: Comparar o grau de ansiedade e estresse em dois grupos populacionais de mulheres adolescentes e sua associação com a gravidez.

Metodologia: Estudo transversal analítico com 125 adolescentes grávidas atendidas no Hospital Geral de Medellín (Colômbia) e 124 adolescentes não grávidas estudantes da Escola Jorge Eliécer Gaitán de Medellín (Colômbia). Os participantes responderam à pesquisa de Hamilton para ansiedade e à pesquisa de Holmes e Rahe para estresse psicossocial.

Resultados: A média de idade foi de 17 anos (intervalo interquartil ou IQR $=2$ ) nas gestantes e 13,5 (IQR = 2) nas estudantes. Nas gestantes, a prevalência de ansiedade foi de $68 \%$ e estresse de $73,6 \%$, enquanto no grupo de não grávidas a prevalência de ansiedade foi de $64 \%(\mathrm{OR}=1,2 ; \mathrm{IC} 95 \% 0,20-2,09 . \mathrm{P}=0,5)$ e do estresse a taxa foi de $40 \%(O R=4,18 ;$ IC $95 \% 2,37-7,41 ; p=<0,01)$. Houve associação entre ter teste positivo para ansiedade e mau relacionamento familiar, consumo de álcool e início da vida sexual, enquanto houve associação entre ter teste positivo para estresse e ser menor de 15 anos, ter relacionamento familiar ruim, mau relacionamento com companheiro, consumo de álcool, início da vida sexual e familiares com transtorno mental.

Conclusões: Não há maior prevalência de ansiedade em mães adolescentes atendidas no Hospital Geral de Medellín em comparação com adolescentes não grávidas do Colégio Jorge Eliécer Gaitán em Medellín (Colômbia). Mais estresse foi encontrado entre as adolescentes grávidas. Relações familiares precárias, consumo de álcool e início da vida sexual foram evidenciados como fatores associados, tanto para ansiedade quanto para estresse.

Palavras-chave: adolescente; ansiedade; estresse psicológico; gravidez na adolescência

\section{INTRODUCCIÓN}

Según la Organización Mundial de la Salud (OMS), la adolescencia es el periodo de la vida cuando un individuo adquiere la capacidad reproductiva y logra un desarrollo psicológico desde la niñez a la edad adulta. Abarca el periodo entre los $12 \mathrm{y}$ 17 años ${ }^{1,2}$. En este suceden cambios importantes, físicos, psicológicos y sociales ${ }^{3}$.

En la actualidad hay más de mil millones de adolescentes en el mundo, de los que el $85 \%$ vive en países en vías de desarrollo, y más de la mitad ha tenido relaciones sexuales antes de los 16 años. Además, el 10\% de los partos acontece en adolescentes ${ }^{4}$. El embarazo no planeado en una adolescente afecta negativamente la vida social, interrumpe el proyecto educativo y afecta la capacidad de trabajar ${ }^{5}$.

Desde el punto de vista médico y social, el embarazo en la adolescencia se considera como situación de riesgo ${ }^{6,7}$ y como problema de salud pública. En este grupo de edad se ha descrito un mayor número de abortos, anemia, infecciones urinarias, vaginosis, bacteriuria asintomática ${ }^{2,3}$, preeclampsia-eclampsia ${ }^{4,8-10}$, malnutri- 
ción materna ${ }^{2}$, parto prematuro ${ }^{2,3,8}$, rotura prematura de membrana ${ }^{2,3}$ y cesárea ${ }^{6,8,9,11}$. Por otra parte, dentro de los embarazos en la población de 16 años o menos, existe un riesgo más elevado para el hijo ${ }^{11}$, que incluye hospitalización en neonatología, defectos del tubo neural, mortalidad perinatal y bajo peso al nacer ${ }^{2,4,6,12,13}$. Igualmente, se ha descrito una mayor frecuencia de alteraciones psiquiátricas en estos hijos ${ }^{14}$.

Según el estudio nacional de salud mental de Colombia $^{15}$, en 2015 el $7.2 \%$ de la población entre 12 y 17 años ha sufrido alguna vez en la vida un trastorno psiquiátrico. De estos se detecta el $4.4 \%$ en el último año, y el 2.5 $\%$, en el último mes. Los trastornos de ansiedad encabezan la lista (50\%), seguidos de la fobia social (48\%), los trastornos del estado de ánimo (24\%), el consumo de bebidas alcohólicas durante la vida (20.4\%) y el de tabaco durante la vida (5.8\%).

La prevalencia mundial de trastornos de ansiedad va del 2.4 al 18.2\%. Los trastornos que siguen en frecuencia a los de ansiedad son los del afecto (1.7-9.6\%). Los factores relacionados con el trastorno de ansiedad son el sexo femenino (razón 3:1) y la edad (adulto joven, promedio 25 años, aunque puede comenzar en la niñez o adolescencia). Otros factores asociados son dificultades económicas, estar separado o viudo, consumo de alcohol, eventos estresantes en la infancia, otras morbilidades y antecedente de enfermedad mental en los padres ${ }^{16-17}$.

El estrés es sinónimo de tensión física o emocional que supera la capacidad de cada individuo, proviene de cualquier situación o pensamiento que genere frustración, rabia o ansiedad. Se considera normal cuando es de poca intensidad y a corto plazo, como el asociado a situaciones de riesgos o inesperadas. Todas las personas sienten estrés agudo en algún momento de la vida, este puede ser positivo y desaparece rápidamente; sin embargo, cuando la causa se perpetúa o la respuesta del individuo es inadecuada o intensa, produce trastornos mentales como ansiedad o depresión, así como alteraciones fisiológicas como hipertensión, diarrea o estreñimiento, gastritis, cefalea, desatención, adinamia, falta de concentración, insomnio y problemas sexuales ${ }^{18}$.

En los adolescentes el estrés se puede manifestar con irritabilidad, cambio de apetito o de peso, trastornos del sueño, aprensión, llanto fácil o temblores, así como por cuatro problemas comunes en la adolescencia: abuso de sustancias, iniciación sexual temprana y embarazo, deserción o problemas escolares y delincuencia ${ }^{17}$.

El objetivo del estudio es comparar los niveles de ansiedad y estrés en dos grupos poblacionales de mujeres adolescentes y su asociación con el embarazo.

\section{METODOLOGÍA}

\section{Diseño}

Estudio transversal analitico que incluyó mujeres adolescentes embarazadas y no embarazadas entre 2008 y 2009.

\section{Población y muestra}

Las adolescentes embarazadas (Grupo 1) fueron seleccionadas entre aquellas atendidas en el servicio de alto riesgo obstétrico (ARO) del Hospital Luz Castro de Gutiérrez (Hospital General de Medellín) ubicado en el noroeste de Colombia, de carácter público, centro de referencia de tercer nivel, que atiende a pacientes afiliados al régimen subsidiado de seguridad social, pertenecientes en su mayoría a estratos socioeconómicos 1-2. Por su parte, las adolescentes no embarazadas (Grupo 2) fueron seleccionadas de los grados sexto a once de bachillerato del Colegio Jorge Eliécer Gaitán, de carácter público, ubicado en el barrio Robledo que pertenece mayormente a estratos socioeconómicos bajos de Medellín.

En ambos grupos la edad de inclusión estuvo entre los 12 y 17 años. Se excluyeron aquellas con problemas cognitivos que impidieron la aplicación del instrumento y que no dieron su consentimiento. La muestra se calculó mediante la fórmula de Joseph Fleiss ${ }^{19}$, con una confianza del $95 \%$, un poder del $80 \%$ y un OR de 2.5 ; teniendo en cuenta que la prevalencia de trastorno de ansiedad y estrés en la población general es variable, se tomó como referencia el 15\%. El tamaño de la muestra quedó distribuido de modo que se ubicaron 125 participantes en el grupo 1, y 124, en el 2. En los dos grupos se realizó muestreo secuencial consecutivo.

\section{Instrumentos de recolección y variables}

Después de la estandarización del protocolo de investigación, la información fue recolectada por el investigador mediante encuesta durante entrevista y diligenciada por las participantes mediante lápiz y papel. En el grupo de embarazadas se realizó en el servicio de hospitalización de alto riesgo obstetrico del Hospital General de Medellín, y en el caso de las no embarazadas, en el aula de clase del Colegio Jorge Eliecer Gaitán de Medellín. Se dispuso de un tiempo de 15 minutos. En la primera parte de la encuesta se indagaban variables sociodemográficas y posteriormente se aplicó el test de Hamilton para ansiedad y la escala de reajuste social para el diagnóstico de estrés y sucesos vitales. 


\section{Escalas de medición y validez}

Para el diagnóstico de ansiedad, se utilizó la escala de Hamilton $^{20}$, una escala semiestructurada, hereroaplicada, que evalúa la gravedad de la ansiedad global. Está compuesta por 14 ítems, siendo 13 referentes a signos y síntomas ansiosos, y el último para valorar el comportamiento. Se diligencia por el entrevistador o entrevistado, con una duración de 10 a 30 minutos. El entrevistador puntúa de 0 a 4 puntos cada ítem, valorando tanto la intensidad como la frecuencia. La puntuación total es la suma de las de los ítems. El rango va de 0 a 56 puntos. Permite obtener, además, dos puntuaciones que corresponden a ansiedad psíquica (ítems 1, 2, 3, 4, 5, 6 y 14) y a ansiedad somática (ítems 7, 8, 9, 10, 11, 12 y 13), y se interpreta de acuerdo con los siguientes puntos de corte: no ansiedad $<5$ puntos, ansiedad leve 6-14 puntos, ansiedad moderada $15-25$ puntos, ansiedad grave $26-40$ puntos y muy grave 41-56 puntos.

Propiedades psicométricas. La escala de Hamilton (HARS, por sus siglas en inglés) es válida y apropiada para uso en la práctica asistencial y en investigación. En el estudio de Lobo et al. ${ }^{21}$ encontraron validez convergente con la escala Montgomery-Åsberg de depresión (MADRS, por sus siglas en inglés), así como con otras pruebas de ansiedad y con mediciones de calidad de vida. Asimismo reportaron excelente consistencia interna, fiabilidad entre observadores y en aplicaciones sucesivas.

Para el diagnóstico de estrés y sucesos vitales, se utilizó la escala de reajuste social (SRRS, por sus siglas en inglés) de Holmes y Rahe ${ }^{22}$, una escala semiestructurada, hereroaplicada, que evalúa la magnitud de eventos vitales que se sospeche que anteceden al inicio de una enfermedad o que agravan el curso de una enfermedad establecida. Está conformada por 43 ítems o situaciones (que abarcaban las áreas de salud, trabajo, hogar/familia, personal/ social y financiera). Se diligencia por el entrevistador o entrevistado, con una duración de 15 a 30 minutos. El entrevistador o el entrevistado marca los eventos que se han presentado en el último año y se suman las puntuaciones asignadas a cada uno. La puntuación total es la suma de las de cada uno de los ítems, el rango va de 0 a 1468 puntos.

Al valor asignado a cada ítem o situación promediado se le llama unidad de cambio de vida (LCU, por sus siglas en inglés) y se refiere al grado de reajuste vital (promedio), que demanda esa situación de forma independiente y ha sido validada al español ${ }^{23-24}$.

Propiedades psicométricas. La escala SRRS presenta adecuadas propiedades psicométricas, con lo que resulta apropiada para su uso en la práctica asistencial y en investigación clínica. La suma de los puntajes de LCU también ha mostrado validez para predecir tanto el inicio como la gravedad de una enfermedad subsecuente. Los valores comprendidos entre 0 y 150 puntos implican que no hay problemas significativos salud, el estrés medio bajo (de 151 a 199 puntos) implica un riesgo de enfermar del $33 \%$, el estrés medio (de 200 a 299 puntos) implica riesgo de enfermar del $50 \%$ y el estrés alto (> de 300 puntos) implica riesgo de enfermar del $80 \%$.

\section{Análisis estadístico}

Se evaluaron las siguientes variables: edad, estrato socioeconómico, nivel de estudio, estado civil, ocupación, planeación del embarazo, deseo de tener más hijos, calidad de las relaciones familiares, calidad de la relación de pareja, consumo de drogas, consumo de alcohol, tabaquismo, conocimiento de métodos anticonceptivos, antecedente de planificación, edad de inicio de vida sexual, número de compañeros sexuales, infecciones de transmisión sexual, así como el puntaje en la escala de Hamilton y la escala de reajuste social.

Para el análisis se utilizó el programa Epidat 3.1 de libre distribución. Se resume la información de las variables continuas mediante la mediana y el rango intercuartílico (RIC) y las variables categóricas como frecuencias absolutas y relativas. Se determinó si había distribución normal en el caso de las variables continuas mediante la prueba de Kolmogorov-Smirnov y se realizó análisis bivariado de dichas variables mediante la $U$ de Mann-Whitney. Finalmente el análisis bivariado de las variables categóricas se realizó mediante chi cuadrado.

\section{Consideraciones éticas}

El proyecto fue aprobado por el Comité de Ética del Hospital General de Medellín. El trabajo fue realizado en la Facultad de Ciencias de la Salud de la Universidad CES, Medellín (Colombia). Las participantes fueron informadas acerca del estudio y las interesadas aceptaron participar de manera voluntaria y las pruebas se aplicaron en compañía de padres o adultos responsables que firmaron consentimiento informado. A las adolescentes que presentaron síntomas de ansiedad y estrés se les sugirió atención especializada.

Este se considera un estudio sin riesgo, según la clasificación planteada en el artículo 11 de la Resolución 8430 de 1993 (expedida por el Ministerio de Salud de Colombia), y se ajusta a la normativa internacional (declaración de Helsinki y a las pautas éticas para la investigación biomédica preparadas por el Consejo de Organizaciones Internacionales de las Ciencias Médicas [CIOMS, por sus siglas en inglés]). 


\section{RESULTADOS}

En el grupo de mujeres embarazadas la mediana de la edad fue de 17 (RIC 2) y en el grupo de estudiantes no embarazadas fue de 13.5 (RIC 2). Las otras características sociodemográficas se describen en detalle en la Tabla 1.
Por otra parte, en el Grupo 1 la mediana para la edad de inicio de relaciones sexuales fue de 15 años (RIC 2) y para el número de compañeros sexuales fue de 1 (RIC 1). En el Grupo 2 la mediana para la edad de iniciación de relaciones sexuales fue 16 (RIC 2) y para el número de compañeros sexuales fue de 0 (RIC 0 ). Asimismo, se realizaron comparaciones entre los grupos 1 y 2 , a partir

Tabla 1. Comparación de las características de las poblaciones de adolescentes en Medellín (Colombia). Grupo 1 Adolescentes en embarazo $(n=125)$ y Grupo 2 Adolescentes no embarazadas $(n=124)$.

\begin{tabular}{|c|c|c|c|}
\hline Variable & Grupo 1, n (\%) & Grupo 2, n (\%) & $\mathbf{p}$ \\
\hline $\begin{array}{l}\text { Estrato } \\
1 \text { y } 2 \\
3 \text { y } 4\end{array}$ & $\begin{array}{c}109(87.2) \\
16(12.8)\end{array}$ & $\begin{array}{l}94(75.8) \\
30(24.1)\end{array}$ & $0.021^{*}$ \\
\hline $\begin{array}{l}\text { Escolaridad } \\
\text { Primaria } \\
\text { Secundaria } \\
\text { Otros }\end{array}$ & $\begin{array}{l}25(20.1) \\
84(67.7) \\
16(12.2)\end{array}$ & $\begin{array}{c}2(1.61) \\
120(96.7) \\
2(1.61)\end{array}$ & $<0.00^{*}$ \\
\hline $\begin{array}{l}\text { Estado civil } \\
\text { Casada } \\
\text { Soltera } \\
\text { Unión libre } \\
\text { Viuda }\end{array}$ & $\begin{array}{c}4(3.2) \\
59(47.2) \\
60(48) \\
2(1.6)\end{array}$ & $\begin{array}{c}0 \\
118(94.3) \\
6(4.8) \\
1(0.8)\end{array}$ & $<0.00^{*}$ \\
\hline $\begin{array}{l}\text { Ocupación } \\
\text { Empleado } \\
\text { Sin empleo } \\
\text { Estudiante } \\
\text { Ama de casa }\end{array}$ & $\begin{array}{c}4(3.2) \\
10(8.0) \\
30(24.1) \\
81(64.7)\end{array}$ & $\begin{array}{c}1(0.8) \\
2(1.6) \\
121(97.5) \\
0\end{array}$ & $<0.00^{*}$ \\
\hline $\begin{array}{l}\text { Relaciones familiares } \\
\text { Buena } \\
\text { Regular } \\
\text { Mala }\end{array}$ & $\begin{array}{l}94(75.2) \\
29(23.2) \\
2(1.6)\end{array}$ & $\begin{array}{l}101(75.2) \\
21(26.2) \\
1(0.8)\end{array}$ & $0.42^{*}$ \\
\hline $\begin{array}{l}\text { Relación de pareja } \\
\text { Buena } \\
\text { Regular } \\
\text { Mala }\end{array}$ & $\begin{array}{c}n=122 \\
85(69.6) \\
16(13.1) \\
21(17.2)\end{array}$ & $\begin{array}{c}n=43 \\
40(93) \\
2(4.6) \\
1(2.3)\end{array}$ & $0.008^{\star}$ \\
\hline $\begin{array}{l}\text { Consumo de alcohol } \\
\text { Sí } \\
\text { No }\end{array}$ & $\begin{array}{c}9(7.2) \\
116(92.8)\end{array}$ & $\begin{array}{c}8(6.4) \\
116(93.5)\end{array}$ & $0.81^{*}$ \\
\hline $\begin{array}{l}\text { Tabaquismo } \\
\text { Sí } \\
\text { No }\end{array}$ & $\begin{array}{c}8(7,2) \\
117(93,6)\end{array}$ & $\begin{array}{c}6(4,8) \\
118(95,1)\end{array}$ & $0.59 *$ \\
\hline $\begin{array}{l}\text { Conocimiento sobre planificación } \\
\text { Sí } \\
\text { No }\end{array}$ & $\begin{array}{l}89(71.2) \\
36(28.8)\end{array}$ & $\begin{array}{l}78(63) \\
46(37)\end{array}$ & $0.19 *$ \\
\hline $\begin{array}{l}\text { ¿Ha planificado? } \\
\text { Sí } \\
\text { No }\end{array}$ & $\begin{array}{c}24(19.2) \\
101(80.8)\end{array}$ & $\begin{array}{c}5(4) \\
119(96)\end{array}$ & $<0.00^{*}$ \\
\hline $\begin{array}{l}\text { Inicio vida sexual } \\
<=13 \text { años } \\
>13 \text { años }\end{array}$ & $\begin{array}{c}6(4.8) \\
119(95.2)\end{array}$ & $\begin{array}{c}112(90.3) \\
12(9.7)\end{array}$ & $<0.00^{*}$ \\
\hline $\begin{array}{l}\text { \# compañeros sexuales } \\
1 \text { a } 2 \\
3 \text { a } 4 \\
5 \text { o más }\end{array}$ & $\begin{array}{c}102(81.5) \\
17(13.7) \\
6(4.8)\end{array}$ & $\begin{array}{c}\mathrm{n}=15 \\
14(93.3) \\
1(6.6) \\
0\end{array}$ & $0.48^{*}$ \\
\hline $\begin{array}{l}\text { ETS } \\
\text { Sí } \\
\text { No }\end{array}$ & $\begin{array}{c}2(1.6) \\
123(98.4)\end{array}$ & $\begin{array}{c}0 \\
124(100)\end{array}$ & $0.49^{*}$ \\
\hline
\end{tabular}

* chi cuadrada de asociación.

ETS: enfermedad de transmisión sexual. 
de las cuales se encontraron diferencias significativas para la edad, inicio de relaciones sexuales y número de compañeros sexuales $(\mathrm{p}<0.01)$.

En el Grupo 1 el 77.7\% tenía más de 15 años y el 71.2\% afirmó tener conocimientos sobre métodos anticonceptivos, pero de las que ya iniciaron relaciones sexuales solo el 19.8\% utiliza algún método de planificación familiar. El 59\% de las adolescentes embarazadas no planeó el embarazo y el 77\% no desea tener más hijos.

Los análisis bivariados mostraron diferencias significativas en estrato socioeconómico, escolaridad, estado civil, ocupación, relación de pareja, planificación previa e inicio de la vida sexual. Con respecto al trastorno de ansiedad, en el Grupo 1 se encontró una prevalencia del 68\%, mientras que en el Grupo 2 del 64\%: se halló que las primeras no sufrieron más trastorno de ansiedad (OR 1.2; IC95\% 0.20-2.09.P=0.5). Los resultados se ilustran en la Tabla 2.

Con respecto al estrés, en el Grupo 1 se encontró una prevalencia del 73.6\%, mientras que en el Grupo 2, del 40\%. Las primeras sufrieron más estrés (OR 4.18; IC95\% 2.37-7.41; $\mathrm{p}=<0.01$ ). Los resultados se ilustran en la Tabla 3.

Hubo asociaciones entre la presencia de ansiedad y estrés, y diversos factores sociodemográficos y clínicos que se presentan en la Tabla 4.

Tabla 2. Frecuencia y niveles de trastorno de ansiedad.

\begin{tabular}{lccc}
\hline & & Casos & Controles \\
\hline Ansiedad (Hamilton) & Rango & n (\%) & n (\%) \\
\hline Normal no ansiedad & $0-5$ & $40(32)$ & $45(36)$ \\
Leve & $6-14$ & $48(38.4)$ & $49(39.2)$ \\
Moderada & $15-25$ & $32(25.6)$ & $24(19.2)$ \\
Grave & $26-40$ & $4(3.2)$ & $7(5.6)$ \\
Muy grave & $41-56$ & $1(0.8)$ & $0(0)$ \\
\hline
\end{tabular}

Tabla 3. Frecuencia y niveles de estrés.

\begin{tabular}{|c|c|c|c|}
\hline & & Casos & Controles \\
\hline Test Holmes-Rahe & Rango & n (\%) & n (\%) \\
\hline No problemas significativos & $0-150$ & $33(26.4)$ & $75(60)$ \\
\hline $33 \%$, estrés medio bajo & 151-199 & $25(20)$ & $16(12.8)$ \\
\hline $50 \%$, estrés medio & $200-299$ & $47(37.6)$ & $29(23.2)$ \\
\hline $80 \%$, estrés alto & $>300$ & $20(16)$ & $5(4)$ \\
\hline
\end{tabular}

Tabla 4. Asociación de la ansiedad y el estrés con diversos factores sociodemográficos y clínicos.

\begin{tabular}{lcccc}
\hline \multicolumn{1}{c}{ Variable } & \multicolumn{1}{c}{ Ansiedad } & Estrés \\
\hline Edad <15 años & OR (IC95\%) & $\mathrm{p}^{*}$ & OR (IC95\%) & $\mathrm{p}^{*}$ \\
Relaciones familiares & $0.75(0.42-1.32)$ & 0.29 & $0.45(0.22-0.89)$ & 0.01 \\
Relaciones de pareja & $3.44(1.49-8.88)$ & 0.00 & $3.22(1.18-10.9)$ & 0.01 \\
Embarazo planeado & $1.54(0.82-2.84)$ & 0.13 & $1.89(1.00-3.73)$ & 0.04 \\
Consumo alcohol & $0.48(0.20-1.13)$ & 0.06 & $0.93(0.33-2.76)$ & 0.89 \\
Consumo drogas & $8.58(1.28-364.2)$ & 0.01 & $4.73(1.00-202.3)$ & 0.01 \\
Consumo cigarrillo & $1.55(0.06-38.51)$ & 0.66 & $0.83(0.03-20.7)$ & 0.75 \\
Inicio vida sexual & $3.09(0.66-29.04)$ & 0.12 & $3.78(0.54-163.8)$ & 0.17 \\
Enfermedad mental & $1.77(1.004-3.13)$ & 0.03 & $2.56(1.32-5.03)$ & 0.00 \\
Familiares con enfermedad mental & $2.23(0.84-6.91)$ & 0.08 & $4.54(1.08-40.4)$ & 0.02 \\
\hline
\end{tabular}

*Para chi cuadrada.

OR: odds ratio, IC95\%: intervalo de confianza 95\%. 


\section{DISCUSIÓN}

En el grupo de madres adolescentes se encontró una prevalencia de ansiedad del $68 \%$, en muchos casos síntomas leves (38.4\%), mientras que los síntomas moderados y graves ocurrieron en el 29.6\%. Un hallazgo similar se reporta en el estudio de Ricardo et al. ${ }^{25}$ quienes encontraron una prevalencia de ansiedad en embarazadas del $40.7 \%$.

En el estudio las malas relaciones familiares, el consumo de alcohol y el inicio de vida sexual fueron factores de riesgo para ansiedad. El citado estudio de Ricardo et al. ${ }^{25}$ evaluó la sintomatología ansiosa de un grupo de 189 mujeres con gestación de alto riesgo y reporta asociación de este síntoma con haber sido víctima de situaciones sociales adversas o haber vivido un desastre natural.

En el grupo de estudiantes de colegio público se encontró una prevalencia de ansiedad del 64\%, también en su mayoría sintomas leves (39.2\%), mientras que los síntomas moderados y graves ocurrieron en el $24.8 \%$. Similar a los reportado por otros autores, con prevalencia que oscila entre el 9 y el $21 \%{ }^{26}$. Los resultados de algunos estudios indican que no todos los adolescentes con ansiedad están limitados de modo importante en su funcionamiento diario.

En el grupo de estudiantes de colegios públicos se encontró una prevalencia de estrés del $40 \%$, la mayoría estrés medio (23.3\%), mientras que el grado alto de estrés ocurrió en el 4\%, de nuevo, similar a los reportados por otros autores con prevalencia del $48,7 \%{ }^{27}$.

En el grupo de madres adolescentes se encontró una prevalencia de estrés del $73.6 \%$, en su mayoría estrés medio (37.6\%), mientras que los síntomas de estrés alto ocurrieron en el 16\%. Un hallazgo similar se reporta en el estudio de Ortiz et al. quienes encontraron una prevalencia de estrés en embarazadas del $37.5 \%{ }^{28}$.

En el estudio se encontró que la edad menor de 15 años, las malas relaciones familiares y de pareja, el consumo de alcohol, el inicio de vida sexual y familiares con enfermedad mental son factores de riesgo para estrés. Algunos estudios reportan que las gestantes con estrés psicosocial tienen 25-60\% más riesgo de prematuridad. Los factores estresantes psicosociales incluyen acontecimientos vitales negativos (muerte o enfermedad crónica de un familiar), catástrofes (desastres, terremotos, terrorismo), eventos crónicos estresantes, depresión, ansiedad en general y específica del embarazo, percepción de inseguridad, discriminación y pobre apoyo social ${ }^{29}$.

En cuanto al conocimientos de métodos anticonceptivos y uso de métodos de planificación, en el estudio se reportan resultados similares a los de Alvarado et al. ${ }^{30}$ en un estudio de 208 mujeres embarazadas donde se encontró que el $55.7 \%$ no había planificado su embarazo.

Es importante intervenir los factores de riesgo específicos para ansiedad y estrés, ya que se ha demostrado que implementando programas de control, destinados especialmente a las adolescentes embarazadas, mejora el pronóstico materno y perinatal ${ }^{31}$. Dichos programas deben contar con seguimiento en el tiempo y evaluación del impacto.

Aunque existe información sobre la magnitud del problema del embarazo en adolescentes en Colombia, los datos son escasos sobre la frecuencia de ansiedad y estrés en madres adolescentes; en el estudio se presentan prevalencias y se describen factores asociados. Como limitación hay que mencionar que el diseño transversal solo permite determinar en un momento preciso los síntomas mentales estudiados. Asimismo, los grupos analizados podrían tener diferencias poblacionales, por ejemplo, la población hospitalaria podría tener mayor riesgo de estrés por ser de alto riesgo obstétrico o por presentar patología concomitante. Finalmente, al tratarse de una muestra por conveniencia, se debe tener cuidado a la hora de generalizar la información.

En conclusión, la ansiedad y el estrés psicosocial son frecuentes en ambos grupos. No se encontró diferencia para la ansiedad, mientras que para el estrés se halló una mayor frecuencia entre el grupo de adolescentes embarazadas con respecto a las estudiantes no embarazadas. Son factores a considerar para la prevención, las malas relaciones familiares, el consumo de alcohol y el inicio de la vida sexual. Se requieren nuevos estudios para confirmar la magnitud del problema en los contextos local y regional.

\section{DECLARACIÓN DE CONFLICTO DE INTERESES}

El autor declara no tener ningún conflicto de intereses. REFERENCIAS

1. Issler JR. Embarazo en la adolescencia. Rev Posgr VIa Cátedr Med 2001;107:11-23.

2. Fernández LS, Carro $E$, Oses $D$, Pérez J. Caracterización del recién nacido en una muestra de gestantes adolescentes. Rev Cubana Obstet Ginecol [Internet] 2004;30 [citado 2020 Nov 18]. Disponible en: http://scielo.sld.cu/scielo.php?script=sci_arttext\&pid=S0138$600 \times 2004000200003 \&$ lng $=$ es.

3. Conde Agudelo A, Belizán JM, Díaz-Rossello JL. Epidemiology of pregnancy in Latin America. 
Acta Obstet Gynecol Scand 2000;79:371-8.

4. Távara Orozco L. Contribución de las adolescentes a la muerte materna en el Perú. Ginecol Obstet 2004;50:111-22.

5. Ortigoza Corona E, Padilla Yasso PY, López Ortiz R. Necesidades educativas en salud perinatal en madres de adolescentes embarazadas. Ginecol Obstet Mex. 2002;70:28-35.

6. Lezcano SA, Vallejos Arce MS, Sodero H. Caracterización del recién nacido en madres adolescentes. Rev Posgr VIa Cátedr Med 2005;149:6-8.

7. Keenan-Miller $\mathrm{D}$, Hammen $\mathrm{CL}$, Brennan PA. Health outcomes related to early adolescent depression. J Adolescent Health 2007;41:256-62.

8. Valdés S, Essien J, Bardales J, Saavedra D, Bardales E. Embarazo en la adolescencia: incidencia, riesgos y complicaciones. Rev Cubana Obstet Ginecol [Internet] 2002;28. [citado 2020 Nov 18]. Disponible en: http://www.bvs.sld.cu/revistas/gin/vol28_2_02/gin04202.htm

9. León JC, Hernández LAI, Hernández AC. Influencia de los factores socioculturales en la morbimortalidad materna y perinatal de adolescentes atendidas en tres estados de la república mexicana. Ginecol Obstet Mex 2002;70:320-7.

10. Cabezas Cruz E. Mortalidad materna y perinatal en adolescentes. Rev Cubana Obstet Ginecol 2002:28:5-10.

11. Pereira LS, Plascencia JL, Ahued RA, García Benítez CQ, Priego PI, Gómez Arteaga C. Morbilidad materna en la adolescente embarazada. Ginecol Obstet Méx 2002;70:270-4.

12. Zelaya MB, Godoy AC, Esperanza LM. Estado nutricional en el embarazo y peso del recién nacido. Rev Posgr VIa Cátedr Med 2003;125:1-6.

13. Balestena JM, Balestena SG. Impacto de la menarquia en los resultados maternos perinatales en la adolescencia. Rev Cubana Obstet Ginecol [Internet] 2005;31(1). [citado $2010 \mathrm{Ag}$ 18]. Disponible en: http://www.bvs. sld.cu/revistas/gin/vol31_1_05/gin02105.htm

14. Rodríguez PL, Hernández J, Reyes A. Bajo peso al nacer: algunos factores asociados a la madre. Rev Cubana Obstet Ginecol [Internet] 2005;31(1). [citado 2020 Nov 18]. Disponible en: http:// www.bvs. sld.cu/revistas/gin/vol31_1_05/gin05105.htm

15. Ministerio de Salud y Protección Social, Colciencias. Encuesta Nacional de Salud Mental 2015, Bogotá: Javegraf [Internet]. [citado 2020 Nov 18]. Disponible en: https://www.saldarriagaconcha. org/wp-content/uploads/2019/01/Encuesta_Nacional_de_Salud_Mental_Tomo_I.pdf

16. Kessler RC, Chiu WT, Demler O, Merikangas KR, Walters EE. Prevalence, severity, and comorbidity of 12-month DSM-IV disorders in the National Comorbidity Survey Replication. Arch Gen Psychiatry 2005;62:617-27.

17. Moreno-Peral P, Conejo-Cerón S, Motrico E, Rodríguez-Morejón A, Fernández A, García-Campayo $J$, et al. Risk factors for the onset of panic and generalised anxiety disorders in the general adult population: a systematic review of cohort studies. J Affect Disord 2014;168:337-48.

18. El estrés y su salud: MedlinePlus enciclopedia médica [Internet]. [citado 2020 Nov 18]. Disponible en: https://medlineplus.gov/spanish/ency/article/003211.htm

19. Fleiss J. Statistical methods for rates and proportions. 2. $^{a}$ ed. Nueva York: Wiley;1981. p. 38-46.

20. Hamilton M. The assessment of anxiety states by rating. Br J Med Psychiat 1959;32:50-5.

21. Lobo A, Chamorro L, Luque A, Dal-Ré R, Badía X, Baró E y el Grupo de Validación en Español de Escalas Psicosométricas (GVPEEP). Validación de las versiones en español de la MontgomeryAsberg Depression Rating Scale y la Hamilton Anxiety Rating Scale para la evaluación de la depresión y de la ansiedad. Med. Clin (Barc) 2002;118:439-9.

22. Holmes TH, Rahe RH. The social readjustment rating scale. J Psychosom Res. 1967;11:213-18.

23. Acuña L, González-García D, Bruner C. La Escala de Reajuste Social de Holmes y Rahe en México: una revisión después de 16 años. Revista Mexicana de Psicología 2012;29:16-32.

24. González J, Revuelta A. La valoración de sucesos vitales: adaptación española de la escala de Holmes y Rahe. Psiquis 1983;4:7-11.

25. Ricardo-Ramírez C, Álvarez-Gómez M, Ocampo-Saldarriaga MV, Tirado-Otálvaro AF. Prevalencia de tamizaje positivo para depresión y ansiedad en gestantes de alto riesgo obstétrico en una clínica de Medellín, entre enero y agosto de 2013: factores de riesgo asociados. Rev Colomb Obstet Ginecol 2015;66:94-102.

26. Graham P, Turk J, Verhulst F. Development and developmental psychopathology. En: Graham P, Turk J, Verhulst F, editores. Child psychiatry: a developmental approach. 3. ${ }^{a}$ ed. Nueva York: Oxford University Press; 1999. p. 172-266.

27. Menéndez C, Montes A, Núñez C, Fernández M, Gamarra T, Buján S. Estrés ambiental y reactividad cardiovascular: la influencia de los acontecimientos vitales estresantes en pacientes hipertensos. Aten Primaria 2002;10:631-7.

28. Ortiz Martínez RA, Castillo A. Relación entre estrés durante el embarazo y nacimiento pretérmino espontáneo. Rev Colomb Psiquiatr 2016;45:75-83.

29. Ibanez G, Charles MA, Forhan A, Magnin G, Thiebaugeorges O, Kaminski M, et al. Depression and anxiety in women during pregnancy and neonatal outcome: data from the EDEN motherchild cohort. Early Hum Dev 2012;88:643-9.

30. Alvarado M, Medina E, Aranda W. El efecto de variables psicosociales durante el embarazo, en el peso y la edad gestacional del recién nacido. Rev Méd Chile 2002;130:561-8.

31. Díaz A, Sanhueza, Yaksic N. Riesgos obstétricos en el embarazo adolescente, estudio comparativo de resultados obstétricos y perinatales con pacientes embarazadas adultas. Rev Chil Obstet Ginecol 2002;67:481-7. 